\title{
Magnetism of atomically thin fcc Fe overlayers on an expanded fcc lattice: $\mathrm{Cu}_{84} \mathrm{Al}_{16}(\mathbf{1 0 0})$
}

\author{
Waldemar A. A. Macedo \\ Laboratório de Física Aplicada, Centro de Desenvolvimento da Tecnologia Nuclear, CNEN, 31270-010 Belo Horizonte, Brazil
}

Fausto Sirotti and Giancarlo Panaccione

Laboratoire pour l'Utilisation du Rayonnement Electromagnetique, CNRS, CEA, MESR, F-94305 Orsay, France

Axel Schatz* and Werner Keune

Laboratorium für Angewandte Physik, Gerhard-Mercator-Universität Duisburg, D47048 Duisburg, Germany

Wagner N. Rodrigues

Departamento de Física, ICEX, Universidade Federal de Minas Gerais, Belo Horizonte, Brazil

\author{
Giorgio Rossi \\ INFM, Dipartimento di Fisica dell' Università di Modena, I-41100 Modena, Italy \\ and Laboratorium für Festkörperphysik, ETH-Zürich, CH-8093, Switzerland
}

(Received 20 October 1997; revised manuscript received 6 April 1998)

\begin{abstract}
We present experimental data on the magnetic properties of atomically thin fcc ( $\gamma$-phase) Fe films (1-6 atomic layer nominal thickness) epitaxially grown on $\mathrm{Cu}_{84} \mathrm{Al}_{16}(100)$ obtained by linear magnetic dichroism in the angular distribution of Fe $3 p$ core photoelectrons excited by linearly polarized synchrotron radiation. The sign and magnitude of the $\mathrm{Fe} 3 p$ photoemission magnetic asymmetry indicates the onset of in-plane ferromagnetism at 2.5(2) monolayer (ML) thickness of $\gamma$-Fe. The Curie temperature is 288(2) K for 4 ML thickness. The magnetic splitting of the Fe $3 p m j$ core hole sublevels is $1.10(2) \mathrm{eV}$, i.e., the same value as measured for a bcc-Fe(100) surface where large surface and near-surface enhanced moments contribute. These results characterize the epitaxial $\gamma$-Fe on $\mathrm{Cu}_{84} \mathrm{Al}_{16}(100)$ as a high-spin ferromagnet for thickness up to $4 \mathrm{ML}$, with an average magnetic moment per iron atom of 2.5(1) $\mu_{B}$. A phase transition occurs between 4 and 5 ML thickness: the magnetic order of the pseudomorphic $\gamma$-Fe film decreases consistently with the breaking into two phases with the deeper layers in a low-spin and/or antiferromagnetic phase and surface restricted ferromagnetism, similar to the case of $\gamma-\mathrm{Fe} / \mathrm{Cu}(100)$. [S0163-1829(98)09833-6]
\end{abstract}

\section{INTRODUCTION}

Achieving an understanding of the properties and behavior of artificial nanostructures obtained by epitaxial growth and other new techniques in the search for unusual properties of materials is one of the dominant trends in physics and materials science nowadays. For magnetic materials, the search for correlation between structure and magnetic properties of metastable phases of transition metals and other nonconventional materials is particularly interesting. Among these metastable phases, fcc Fe $(\gamma$-Fe $)$ has attracted much attention from theoretical and experimental groups. The relevance of this fact lies in the possibility of studying the magnetic properties of iron in metastable phases where it is expected that magnetovolume effects determine the groundstate configuration. ${ }^{1-3}$ Theoretical calculations of the ground-state of bulk $\gamma$-Fe predict a nonmagnetic phase, an antiferromagnetic phase, and two ferromagnetic phases, one with a small magnetic moment of $1.1 \mu_{B}$, and one with a high magnetic moment of $2.5 \mu_{B}$ per atom. ${ }^{1-3}$ The lattice parameter, i.e., the volume available for the iron atoms, determines the ground state of $\gamma$-Fe and a magnetovolume instability is predicted at $3.66 \AA$ lattice spacing (or $12.25 \AA^{3}$ volume) inducing a first-order phase transition from the antiferromagnetic and low-spin phases to the ferromagnetic high-spin state. ${ }^{1-3}$ Moreover, within the low-spin and in the high-spin states, the fcc Fe magnetic moment is predicted to increase monotonically with increasing lattice spacing. ${ }^{1-4}$ Experimentally, bulk fcc Fe is stable only at elevated temperatures $\left(>910^{\circ} \mathrm{C}\right)$ as a paramagnetic metal and can be stabilized at lower temperatures as coherent precipitates in $\mathrm{Cu}$ and $\mathrm{CuAl}$ matrixes ${ }^{5,6}$ or by epitaxy onto suitable fcc substrates. ${ }^{7-17} \mathrm{Cu}$ has a lattice parameter of $3.61 \AA$ and therefore a volume per atom of $11.76 \AA^{3}$ that would correspond to a $\gamma$-Fe antiferromagnetic or low-spin phase. ${ }^{1}$ Ferromagnetic order of $\gamma$-Fe was observed first for films grown by electrolytic methods on $\mathrm{Cu}(110)$ (Ref. 8) and for ultrathin films grown by molecular-beam epitaxy (MBE) methods on $\mathrm{Cu}(111)$. The experimental evidence of the stabilization of the high-spin phase, with a measured moment of $2.6 \mu_{B}$, was obtained for $\gamma$-Fe as grown on $\mathrm{CuAu}$ substrates. ${ }^{11}$ The growth of $\gamma$-Fe on $\mathrm{Cu}(100)$ is in fact pseudomorphic: an expansion of the lattice perpendicular to the surface can lead the system to the magnetovolume instability. The complexity of the experimental results obtained on $\mathrm{Fe} / \mathrm{Cu}(100)$ is understood as a consequence of the exact structure taken by the $\gamma$-Fe as a function of thickness, deposition temperature, and interdiffusion with the substrate. A complex correspondence of magnetism and structure has been recently established. ${ }^{18-28}$ Nondistorted fcc $\mathrm{Fe} / \mathrm{Cu}(100)$ shows antifer- 
romagnetic order, while an expansion of the Fe lattice perpendicular to the surface can lead to "fcc-like" Fe in the ferromagnetic state. ${ }^{19-26}$

In this paper we present surface sensitive magnetometric results obtained on epitaxial ultrathin $\mathrm{Fe}$ films grown on $\mathrm{Cu}_{84} \mathrm{Al}_{16}(100)$, a fcc substrate chosen in order to favor the stabilization of the "high spin" phase of $\gamma$-Fe since it has a lattice parameter $1 \%$ larger than pure $\mathrm{fcc}-\mathrm{Cu}$ and therefore a $12.15 \AA^{3}$ volume, tuned to that predicted for the high-spin fcc Fe. The magnetism of the fcc iron epitaxial layers has been probed by linear magnetic dichroism in the angular distribution of photoelectron intensity (LMDAD) of the $\mathrm{Fe}$ $3 p$ core levels. This novel method ${ }^{29-32}$ allows us to analyze, in a fairly independent way, ${ }^{33,34}$ the magnetic order of the fcc iron surfaces via the magnitude of the LMDAD asymmetry, and the relative changes of the local magnetic moment of the $\mathrm{Fe}$ atoms via the changes of the energy splitting of the magnetic sublevels of the Fe $3 p$ core hole. This is a photoelectron spectroscopy experiment: the magnetic information that is derived is an average of the contributions of the top layers, weighted by the photoelectron escape depth.

\section{EXPERIMENT}

Epitaxial Fe overlayers (1 to $6 \mathrm{ML}$ thick) were grown under MBE conditions by $e$-beam evaporation of a highpurity $\mathrm{Fe}$ wire $(5 N)$ onto a clean surface of a $\mathrm{Cu}_{84} \mathrm{Al}_{16}(100)$ single crystal that presents a lattice parameter of $3.65 \AA$, as determined by x-ray diffraction. Both growth and measurements were performed in an ultrahigh vacuum system with a base pressure of $1 \times 10^{-10} \mathrm{mbar}$ connected to the SU7 undulator beam line at the SuperAco storage ring at Orsay. The energy of the linearly polarized undulator radiation was selected at $170 \mathrm{eV}$ in order to obtain $\mathrm{Fe} 3 p$ photoelectrons with kinetic energies such to give a minimum probing depth $\lambda=5(1) \AA$. This means that about $30 \%$ of the photoemission intensity originates from the surface and that the subsurface contributions are weighted by $e^{-d / \lambda}$, where $d$ is the distance of the deeper layer from the vacuum interface. The $\mathrm{Cu}_{84} \mathrm{Al}_{16}(100)$ surface was cleaned by cycles of $\mathrm{Ar}^{+}$sputtering and annealing. Reproducible results were obtained for 30 min $\mathrm{Ar}^{+}$sputtering at $1.5 \mathrm{kV}$ at room temperature (RT) followed by $20 \mathrm{~min}$ annealing at $650 \mathrm{~K}$ : sharp $p(1 \times 1)$ lowenergy electron diffraction (LEED) patterns for $75 \mathrm{eV}$ primary electrons were obtained. No traces of $\mathrm{C}$ were measured, but a small oxide signal on the $\mathrm{Al} 2 p$ core level photoemission peak indicated the presence of some oxidized aluminum at the surface. The Fe evaporation rate was 0.3 $\AA /$ min, as determined by a calibrated quartz oscillator, and the residual gas pressure during the Fe evaporation was always better than $8 \times 10^{-10} \mathrm{mBar}$. Two growth conditions were explored: the $\mathrm{Cu}_{84} \mathrm{Al}_{16}$ substrate was held at $\mathrm{RT}$ and at $150 \mathrm{~K}$ during iron deposition. The magnetic measurements were done by LMDAD using the same experimental setup and chiral geometry as described elsewhere. ${ }^{35}$

We have measured the $\mathrm{Fe} 3 p$ core level from the $\mathrm{Fe}$ monolayers and mirror experiments were achieved by aligning the in-plane magnetization of the $\mathrm{Fe}$ surface via an external field up or down along the vertical direction, perpendicular to the scattering plane. The magnetic (LMDAD) asymmetry is defined as $A_{L M D A D}$

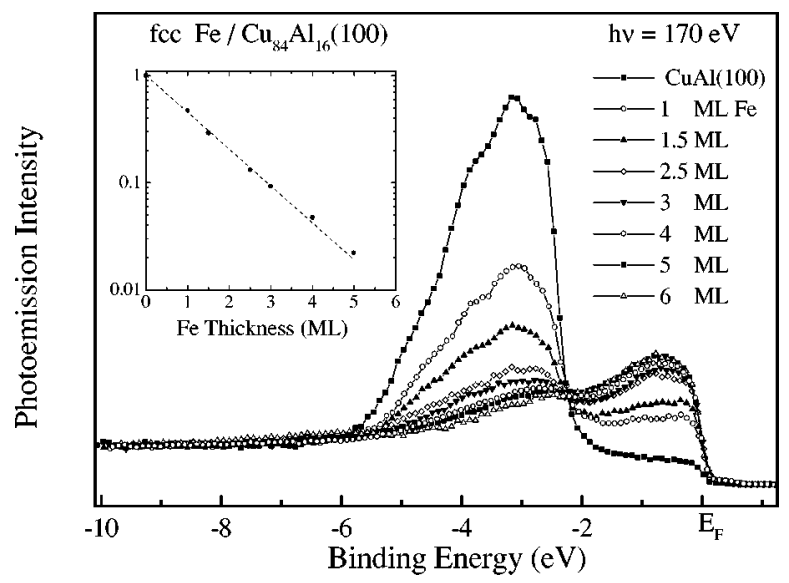

FIG. 1. SR-PES valence band spectra as a function of the Fe coverage, for the $\mathrm{Fe} / \mathrm{Cu}_{84} \mathrm{Al}_{16}(100)$ system. Inset: valence band intensity decay. The result of the fitting in the inset indicates a layerby-layer growth of the Fe films.

$=\left[\left(I_{u p}-I_{\text {down }} / I_{\text {up }}+I_{\text {down }}\right)\right]$, where $I_{\text {up }}\left(I_{\text {down }}\right)$ were the photoelectron spectral intensities obtained with the sample magnetization in the upward ( $u p)$ or downward (down) directions. In this geometry $A_{L M D A D}$ is nonzero only if the surface magnetization has a sizable in-plane component. The sample position and the photon energy were kept fixed in order to avoid photoelectron diffraction effects. ${ }^{36}$

\section{RESULTS AND DISCUSSION}

The epitaxial growth of ultrathin $\mathrm{Fe}$ layers on the $\mathrm{Cu}_{84} \mathrm{Al}_{16}(100)$ surface at $\mathrm{RT}$ and $150 \mathrm{~K}$ was confirmed by LEED. The LEED patterns from the Fe films showed a sharp $p(1 \times 1)$ structure for RT growth and the same pattern with somewhat broadened spots for growth at $150 \mathrm{~K}$ with the same spacing as the fcc substrate. Samples grown at RT exhibited Al diffusion from the substrate through the $\mathrm{Fe}$ overlayers. These samples did not show magnetic dichroism either at RT or after cooling down to $150 \mathrm{~K}$. The lowtemperature grown films were magnetically ordered and no interdiffusion was observed within the time of the experiments. We restrict the analysis and discussion to the magnetic $\gamma$-Fe layers grown at $150 \mathrm{~K}$.

Figure 1 presents the valence-band spectra taken at 170 $\mathrm{eV}$ photon energy for the clean $\mathrm{Cu}_{84} \mathrm{Al}_{16}(100)$ substrate and for different $\mathrm{Fe}$ coverages, deposited and measured at $150 \mathrm{~K}$. The spectrum of the clean substrate is dominated by the $\mathrm{Cu} 3 d$ band between 5 and $2.5 \mathrm{eV}$ below the Fermi level. In the spectra of the overlayers, the intensity within $1.5 \mathrm{eV}$ from the Fermi level is mostly due to the $3 d$ band of iron. The inset shows the intensity decay of the $\mathrm{Cu} 3 d$ band of the substrate as a function of the $\mathrm{Fe}$ coverage. The exponential decay indicates that the $\gamma$-Fe films cover the substrate uniformly.

Figure 2 shows the Fe $3 p$ LMDAD for 4 and 5 ML $\gamma$-Fe on $\mathrm{Cu}_{84} \mathrm{Al}_{16}(100)$, prepared and measured at $150 \mathrm{~K}$ with linearly polarized, monochromatic synchrotron radiation of $170 \mathrm{eV}$. The LMDAD spectra indicate in-plane ferromagnetism at these coverages. Figure 3 shows the evolution of $A_{L M D A D}$ with Fe thickness on $\mathrm{Cu}_{84} \mathrm{Al}_{16}(100)$. Up to $2 \mathrm{ML}$, no magnetic order is observed in the direction specified by 


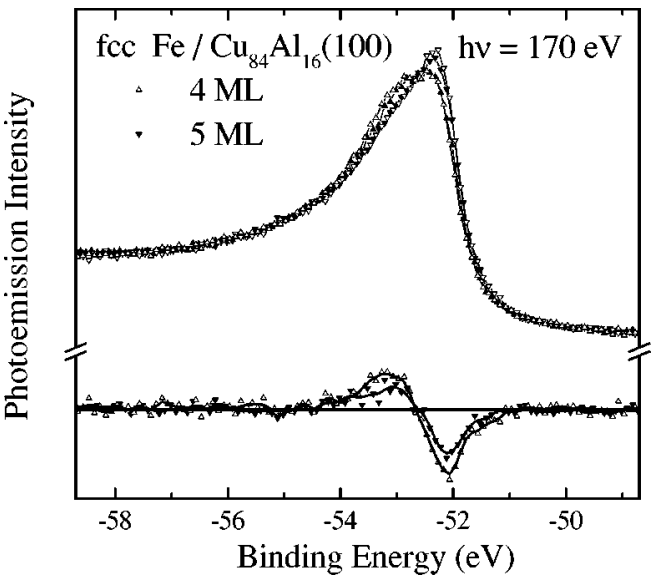

FIG. 2. Fe $3 p$ LMDAD spectra for 4 and 5 ML fcc $\mathrm{Fe} / \mathrm{Cu}_{84} \mathrm{Al}_{16}(100)$ prepared and measured at $150 \mathrm{~K}$. The existence of magnetic asymmetries indicates in-plane ferromagnetism for the 4 and 5 ML-thick $\gamma$-Fe films. Up (down) triangles refer to upward (downward) direction of the sample magnetization with respect to the photoemission plane. Empty (full) symbols refer to the 4 (5 ML) thick iron layer.

the external applied field (in-plane and perpendicular to the photoemission plane). In-plane ferromagnetism appears at $2.5 \mathrm{ML}$ and $A_{L M D A D}$ reaches a maximum value for 3-4 ML. Assuming that below 2.5 ML our Fe films present perpendicular anisotropy, this result would indicate a lower thickness threshold for the reorientation transition of the ferromagnetic easy axis than reported in the case of a $\mathrm{Cu}(100)$ substrate. ${ }^{20,22,25,37}$

The Curie temperature of the in-plane magnetized ultrathin $\gamma$-Fe phase has been determined for 4 ML thickness by measuring $A_{L M D A D}$ as a function of temperature, as shown in Fig. 4. The experiment was performed by varying the temperature of the $150 \mathrm{~K}$ deposited sample between 150 and 300 $\mathrm{K}$. The data are fitted by a function describing the power-law dependence of the order parameter of ferromagnetism in the neighborhood of the Curie temperature $T_{C} .{ }^{38}$ The fit is obtained by maximizing the function $\log \left[1-\left(T / T_{C}\right)\right]$ in the region where $\log (\mathrm{LMDAD})$ vs $\log \left[1-\left(T / T_{C}\right)\right]$ is linear. This procedure yields an exponent $\beta=0.212(5)$ for the power law $\left[1-\left(T / T_{C}\right)\right]^{\beta}$ and a Curie temperature $T_{C}=288(5) \mathrm{K}$.

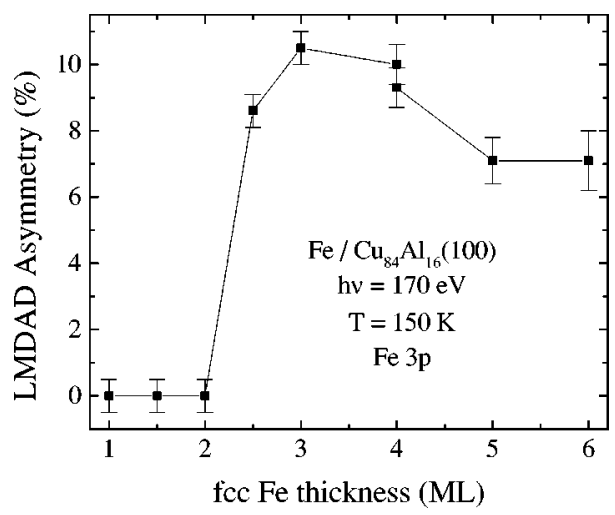

FIG. 3. Fe $3 p$ LMDAD asymmetry for increasing Fe coverage on $\mathrm{Cu}_{84} \mathrm{Al}_{16}(100)$, indicating the onset of in-plane magnetization at 2.5 ML Fe and the phase transition between 4 and $5 \mathrm{ML}$, with $35 \%$ reduction of magnetization.
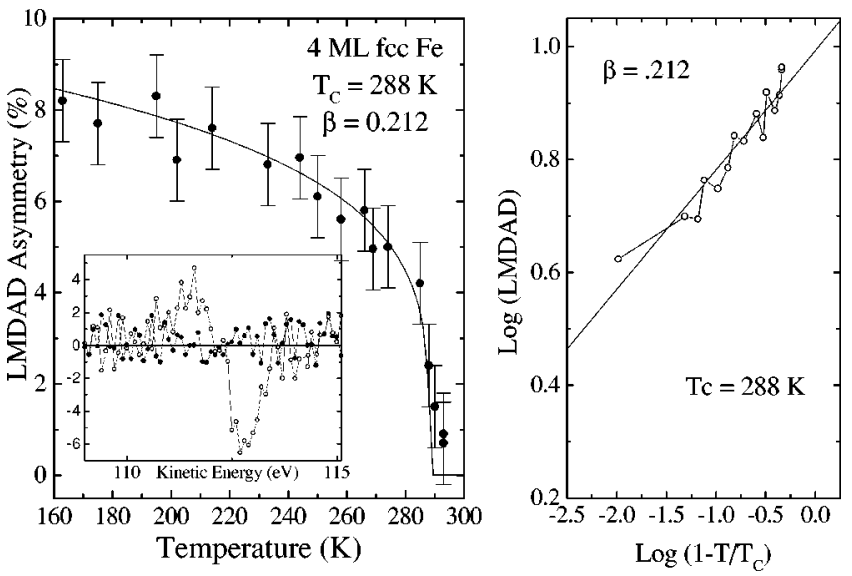

FIG. 4. Temperature dependence of the Fe $3 p$ LMDAD for 4 ML fcc Fe on $\mathrm{Cu}_{84} \mathrm{Al}_{16}(100)$. The data are fitted by the power law $\left[1-\left(T / T_{C}\right)\right]^{\beta}$. The inset shows the LMDAD asymmetries at $150 \mathrm{~K}$ (empty circles) and $300 \mathrm{~K}$ (dots). The second panel shows the fitting procedure based on the power-law hypothesis, according to Dürr et al. (Ref. 40).

Similar $T_{C}$ values have been reported for $\mathrm{Fe} / \mathrm{Cu}(100)$ (Refs. 26 and 15$)$ and $\mathrm{Fe} / \mathrm{CuAu}(111) .{ }^{11}$ Our $\beta$ value agrees well with that predicted by the two-dimensional $X Y$ model $^{39}$ and with values measured on $\mathrm{Fe} / \mathrm{Au}(100)$ (Ref. 40) and on $\mathrm{Fe} / \mathrm{W}(100){ }^{41}$ This extrapolation method for $T_{C}$ allows for the existence of a deviation from the power law above a certain temperature. ${ }^{40,42}$ The deviation is connected to the low dimensionality of the system: in quasi-two-dimensional systems the spin fluctuations related to the phase transition are very important and affect a larger temperature range near $T_{C}$ than in bulk ferromagnetism. By reaching $T_{C}$ from below, the magnetization may not vanish because of the formation of short-range ordered spin clusters that can show different $T_{C}$ values, depending on their size. ${ }^{43}$ The spread of the measured $\mathrm{A}_{L M D A D}$ values near $T_{C}$ provides evidence for a limited coherence length that is connected to the high density of defects in the low-temperature grown layer. The possible onset of interdiffusion at the interface, when annealing at room temperature, may have a direct consequence on the deviation from the power-law behavior.

The analysis of the energy splitting of the Fe $3 p$ core level can give insight on the local magnetic moment of the iron atoms in the epitaxial films. In Fig. 5 we compare the $\mathrm{Fe}$ $3 p$ LMDAD splitting of $3 \mathrm{ML}$ fcc-Fe on $\mathrm{Cu}_{84} \mathrm{Al}_{16}(100)$ $[\Delta E=1.10(2) \mathrm{eV}]$ of a standard bcc-Fe(100) surface. The energy width of the dichroism spectrum represents the energy splitting of the $J=\frac{3}{2}$ multiplet due to the exchange interaction for the $3 p$ core hole and the spin-polarized valence band. The splitting reflects therefore the value of the magnetic moment of the excited atom, i.e., a local property. ${ }^{30,32}$ Recent experiments on $\mathrm{Fe}-\mathrm{Co}$ and $\mathrm{Fe}-\mathrm{Ni}$ surface alloys ${ }^{34}$ have demonstrated that the width of the $3 p$ core level LMDAD spectra is proportional to the local magnetic moment. This means that sizable changes of the iron local magnetic moment are reflected in changes of the splitting energy of the core hole magnetic sublevel, and consequently on the width of the LMDAD spectrum. Roughly speaking, the width of the LMDAD spectrum for the predicted low-spin ferromagnetic phase with $1.1 \mu_{B}$ should be reduced to one- 


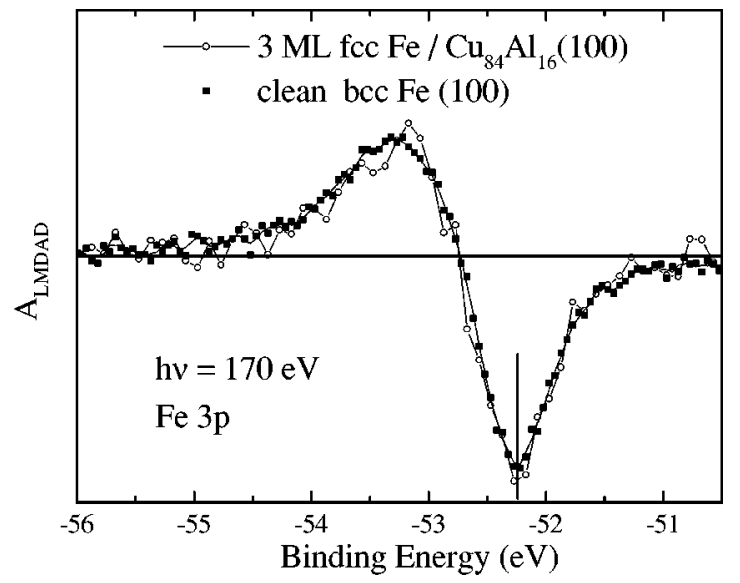

FIG. 5. Fe $3 p$ LMDAD for $4 \mathrm{ML}$ fcc Fe on $\mathrm{Cu}_{84} \mathrm{Al}_{16}(100)$ (open circles) and for bcc-Fe(100) (filled circles). The bcc-Fe(100) spectrum represents the average of a surface enhanced moment of $\sim 3 \mu_{B}$ and subsurface and bulk contributions of $\sim 2.2 \mu_{B}$. According to an escape depth $\lambda=5$ (1) $\AA, \sim 30 \%$ of the photoemission intensity originates from the surface and the LMDAD lineshape of bcc- $\mathrm{Fe}(100)$ is equivalent to that of a homogeneous $\mathrm{Fe}$ film with $\sim 2.5 \mu_{B}$.

half of the width of the LMDAD spectrum for bulk bcc Fe $\left(2.2 \mu_{B}\right)$. From Fig. 5 we observe that the width of the LMDAD spectrum of $3 \mathrm{ML} \gamma-\mathrm{Fe} / \mathrm{Cu}_{84} \mathrm{Al}_{16}(100)$ and that for the $\alpha$-Fe(100) are identical within experimental accuracy. A discussion of the LMDAD signal of bcc-Fe is needed at this stage: the reported LMDAD spectra were obtained in highly surface sensitive conditions, which implies that the measured LMDAD line shapes represent the average of surface, subsurface, and substrate contributions. ${ }^{34}$ The width of the reference bcc $\mathrm{Fe}(100)$ surface spectrum is determined by the surface enhanced moment, the subsurface, and the bulk moments averaged with relative weights set by the surface sensitivity of the measurement. A numerical simulation of the $\mathrm{Fe}(100)$ LMDAD spectrum, based on the hypothesis of linear dependence of the LMDAD width upon the magnetic moment and on an escape depth of $\lambda=5 \AA$, gives an average value of the magnetic moment of the sampled bcc iron layers of $2.5(1) \mu_{B}$. The enhanced values of the magnetic moment at the bcc $\mathrm{Fe}(100)$ surface and subsurface layers have been taken from theory $\left(2.97 \mu_{B}\right.$ for the surface).$^{44}$ It appears from Fig. 5 that the magnetic moment of the iron atoms in 3 ML $\gamma-\mathrm{Fe} / \mathrm{Cu}_{84} \mathrm{Al}_{16}(100)$ is of the same value. If we make the hypothesis that the magnetic moment of the 3 ML-thick $\gamma$-Fe film is uniform through the layer, then our data are consistent with the formation of the theoretical high-spin ferromagnetic phase characterized by a magnetic moment of 2.5(1) $\mu_{B}$, and with the experimental value from Gradmann and Isbert ${ }^{11}$ larger than in bulk $\alpha$-Fe. In the high-spin state of the $\gamma$-phase, iron is a strong ferromagnet, with an almost full majority $3 d$ band. In these conditions, the surface enhancement of the magnetic moment should be limited to a $10 \%$ effect at most, likewise in the case of hcp or bcc cobalt.

Above $4 \mathrm{ML}$, the LMDAD asymmetry is reduced by $35 \%$, as can be seen in Fig. 2. We could not check structural changes on our samples grown on $\mathrm{Cu}_{84} \mathrm{Al}_{16}(100)$, but the reduction of the LMDAD asymmetry signal is consistent with a picture known from (RT grown) $\mathrm{Fe} / \mathrm{Cu}(100)$ : by in- creasing the Fe thickness, the deeper Fe layers undergo a transformation from a distorted fcc structure towards a more compact undistorted one..$^{19,22,26,27}$ If the top layers remain in the perpendicularly expanded, large volume structure, then a magnetovolume instability in the vertical direction may explain the reduction of LMDAD asymmetry (fewer layers in the high spin state) as well as the permanence of a high magnetic moment near the surface. The dense deep layers could be in the antiferromagnetic state at sufficiently low temperature (paramagnetic at $150 \mathrm{~K}$ ) while the surface/nearsurface layers would remain in the high-spin ferromagnetic state with magnetic moments of $2.5(1) \mu_{B}$. For $\mathrm{Fe} / \mathrm{Cu}_{84} \mathrm{Al}_{16}(100)$, the formation of an antiferromagnetically ordered phase in the deep layers for samples thicker than 4 ML is compatible with the LMDAD results, since it would add a nondichroic spectral contribution with a reduced total photoemission peak width (due to the reduced magnetic moment of $\mathrm{Fe}$ atoms in the antiferromagnetic phase) to the surface (ferromagnetic) dominated LMDAD spectrum. This contribution cannot severely modify the line shape of the surface sensitive $\mathrm{Fe} 3 p$ spectra, but can certainly reduce the $A_{L M D A D}$. On the other hand, if the observed reduction of magnetization for thicknesses larger than $4 \mathrm{ML}$ was due to a transition of the whole $\gamma$-Fe film to a low-spin ferromagnetic phase, as suggested by Durrand et al. for $\mathrm{Fe} / \mathrm{Cu}(111),{ }^{45}$ then a large reduction of the LMDAD splitting should be observed, which is not the case.

\section{CONCLUSIONS}

In conclusion, we have observed in-plane ferromagnetism in ultrathin fcc- $\mathrm{Fe}(100)$ layers on $\mathrm{Cu}_{84} \mathrm{Al}_{16}(100)$ for thickness between 2.5 and $6 \mathrm{ML}$, which were prepared and measured at $150 \mathrm{~K}$. The magnetization is oriented in plane at lower coverage than reported in the studies of $\mathrm{Fe} / \mathrm{Cu}(100)$, perhaps as a consequence of the laterally expanded $\mathrm{Cu}_{84} \mathrm{Al}_{16}(100)$ lattice, which reduces the need of expanding the interlayer distance perpendicularly in order to reach the favorable atomic volume for the high-spin ferromagnetic phase, and consequently reduces the perpendicular anisotropy. The ferromagnetic fcc-Fe(100)/Cu $\mathrm{Cu}_{84} \mathrm{Al}_{16}(100)$ phase has an average magnetic moment of the order of $2.5 \mu_{B}$, as deduced by comparison with the data from a clean $\alpha$ - $\mathrm{Fe}(100)$ surface, and has a Curie temperature close to room temperature for $4 \mathrm{ML}$ thickness. A phase transition is observed between 4 and 5 ML: the order parameter is suddenly reduced but the magnetic splitting of the top layers remains basically unchanged. This can be understood by analogy with the behavior established for $\gamma-\mathrm{Fe} / \mathrm{Cu}(100)$ films of similar thickness that exhibit antiferromagnetically ordered (or paramagnetic) deep layers and surface restricted ferromagnetism.

\section{ACKNOWLEDGMENTS}

W.A.A.M. and W.N.R. acknowledge the hospitality at LURE during the experiments and the financial support of the Brazilian Agencies CAPES, CNPq, and FAPEMIG. A.S. and W.K. aknowledge financial support by the Deutsche Forschungsgemeinschaft (SFB166). G.R. acknowledges friendly support by H. C. Siegmann. 
*Present address: Institut für Metallphysik und Nukleare Festkörperphysik, Technische Universität Braunschweig, 38106 Braunschweig, Germany.

${ }^{1}$ V. L. Moruzzi, P. M. Marcus, and J. Kübler, Phys. Rev. B 39, 6957 (1989), and references therein.

${ }^{2}$ M. Uhl, L. M. Sandrastskii, and J. Kübler, J. Magn. Magn. Mater. 103, 314 (1992).

${ }^{3}$ T. Kraft, P. M. Marcus, and M. Scheffler, Phys. Rev. B 49, 11511 (1994).

${ }^{4}$ D. Guenzburger and D. E. Ellis, Phys. Rev. B 51, 12519 (1995).

${ }^{5}$ J. B. Newkirk, Trans. AIME 209, 1214 (1957).

${ }^{6}$ W. Keune, T. Ezawa, W. A. A. Macedo, U. Glos, and K. P. Schletz, Physica B 161, 269 (1989).

${ }^{7}$ W. A. Jesser and J. M. Mathews, Philos. Mag. 15, 1097 (1967); 17, 595 (1968).

${ }^{8}$ J. G. Wright, Philos. Mag. 24, 217 (1971).

${ }^{9}$ U. Gradmann and P. Tillmanns, Phys. Status Solidi A 44, 539 (1977).

${ }^{10}$ W. Kümmerle and U. Gradmann, Solid State Commun. 24, 33 (1977); Phys. Status Solidi A 45, 171 (1978).

${ }^{11}$ U. Gradmann and H. O. Isbert, J. Magn. Magn. Mater. 15-18, 1109 (1990).

${ }^{12}$ W. A. A. Macedo and W. Keune, Phys. Rev. Lett. 61, 475 (1988), and references therein.

${ }^{13}$ R. Rochow, C. Carbone, Th. Dodt, F. P. Johnen, and E. Kisker, Phys. Rev. B 41, 3426 (1990).

${ }^{14}$ W. A. A. Macedo, W. Keune, and R. D. Ellerbrock, J. Magn. Magn. Mater. 93, 552 (1991).

${ }^{15}$ P. Xhonneux and E. Courtens, Phys. Rev. B 46, 556 (1992).

${ }^{16}$ F. Baudelet, M.-T. Lin, W. Kuch, K. Meinel, B. Choi, C. M. Schneider, and J. Kirschner, Phys. Rev. B 51, 12563 (1995).

${ }^{17}$ W. A. A. Macedo, F. Sirotti, A. Schatz, D. Guarisco, G. Panaccione, and G. Rossi, J. Magn. Magn. Mater. 179-181, 1262 (1998).

${ }^{18}$ D. J. Keavney, D. F. Storm, J. W. Freeland, I. L. Gricorov, and J. C. Walker, Phys. Rev. Lett. 74, 4531 (1995).

${ }^{19}$ R. D. Ellerbrock, A. Fuest, A. Schatz, W. Keune, and R. A. Brand, Phys. Rev. Lett. 74, 3053 (1995); W. Keune, A. Schatz, R. D. Ellerbrock, A. Fuest, K. Wilmers, and R. A. Brand, J. Appl. Phys. 74, 4265 (1996).

${ }^{20}$ S. Müller, P. Bayer, C. Reischl, K. Heinz, B. Feldmann, H. Zillgen, and M. Wuttig, Phys. Rev. Lett. 74, 765 (1995).

${ }^{21}$ S. Müller, P. Bayer, A. Kinne, P. Schmailzl, and K. Heinz, Surf. Sci. 322, 21 (1995).

${ }^{22}$ Dongqi Li, M. Freitag, J. Pearson, Z. Q. Qiu, and S. D. Bader, Phys. Rev. Lett. 72, 3112 (1994).

${ }^{23}$ M. T. Kief and W. F. Egelhoff, Jr., Phys. Rev. B 47, 10785 (1993).
${ }^{24}$ H. Magnan, D. Chandesris, B. Villete, O. Heckmann, and J. Lecante, Phys. Rev. Lett. 67, 859 (1991).

${ }^{25}$ D. P. Pappas, K. P. Kämper, and H. Hopster, Phys. Rev. Lett. 64, 3179 (1990).

${ }^{26}$ M. Wuttig, B. Feldmanm, and T. Flores, Surf. Sci. 331-333, 659 (1995).

${ }^{27}$ J. Thomassen, F. May, B. Feldmann, M. Wuttig, and H. Ibach, Phys. Rev. Lett. 69, 3831 (1992).

${ }^{28}$ Ch. Wursch, C. H. Back, L. Burgi, U. Ramsperger, A. Vaterlaus, U. Maier, D. Pescia, P. Politi, M. G. Pini, and A. Rettori, Phys. Rev. B 55, 5643 (1997).

${ }^{29}$ Ch. Roth, F. U. Hillebrecht, H. Rose, and E. Kisker, Phys. Rev. Lett. 70, 3479 (1993); Solid State Commun. 86, 647 (1993).

${ }^{30}$ G. Rossi, F. Sirotti, N. Cherepkov, F. Combet Farnoux, and G. Panaccione, Solid State Commun. 90, 557 (1994); G. Rossi, F. Sirotti and G. Panaccione, in Core Level Spectroscopies for Magnetic Phenomena: Theory and Experiment, Vol. 345 of NATO Advanced Study Institute, Vol. 345 Series B, edited by P. Bagus, G. Pacchiomi, and F. Parmigiani (Plenum, New York, 1995).

${ }^{31}$ N. A. Cherepkov, Phys. Rev. B 50, 13813 (1994).

${ }^{32}$ F. Sirotti, G. Panaccione, and R. Rossi, Phys. Rev. B 52, 17063 (1995).

${ }^{33}$ G. Panaccione, F. Sirotti, E. Narducci, and G. Rossi, Phys. Rev. B 55, 389 (1997); Surf. Sci. 377-379, 445 (1997).

${ }^{34}$ M. Liberati, thesis Univerità di Modena, Italy 1997; M. Liberati, G. Panaccione, F. Sirotti, P. Prieto, and G. Rossi (unpublished).

${ }^{35}$ F. Sirotti and G. Rossi, Phys. Rev. B 49, 15682 (1994).

${ }^{36}$ F. U. Hillebrecht, H. B. Rose, T. Kinoshita, Y. U. Idzerda, G. van der Laan, R. Denecke, and L. Ley, Phys. Rev. Lett. 75, 2883 (1995).

${ }^{37}$ D. P. Pappas, K.-P. Kämper, and H. Hopster, Phys. Rev. Lett. 64, 3179 (1990).

${ }^{38}$ A. Aharoni, Introduction to the Theory of Ferromagnetism (Clarendon, Oxford, 1996).

${ }^{39}$ S. T. Bramwell and P. C. W. Holdsworth, Phys. Rev. B 49, 8811 (1994); J. Phys.: Condens. Matter 5, 53 (1993).

${ }^{40}$ W. Dürr, M. Taborelli, O. Paul, R. Germar, W. Gudat, D. Pescia, and M. Landolt, Phys. Rev. Lett. 62, 206 (1989).

${ }^{41}$ H. J. Elmers et al., J. Appl. Phys. 79, 4984 (1996).

${ }^{42}$ D. L. Connely, J. S. Loomis, and D. E. Mapoter, Phys. Rev. B 3, 924 (1971).

${ }^{43}$ H. C. Siegmann, J. Phys.: Condens. Matter 4, 8935 (1992).

${ }^{44}$ C. S. Wang and A. J. Freeman, Phys. Rev. B 24, 4364 (1981); O. Eriksson, G. W. Fernando, R. C. Albers, and A. M. Boring, Solid State Commun. 78, 801 (1991); S. R. Chubb and W. E. Pickett, Phys. Rev. B 38, 10227 (1988).

${ }^{45}$ O. Durrand, J. M. George, J. R. Childress, S. Lequien, A. Schuhl, and A. Fert, J. Magn. Magn. Mater. 121, 140 (1993). 